\title{
COEFFICIENT ESTIMATES FOR EXPONENTIAL SERIES
}

\section{W. T. SLEDD}

ABSTRACT. Results are obtained which relate the size of the coefficients $a_{n}$ of an exponential series $f(x)=\Sigma_{n=0}^{\infty} a_{n} \epsilon^{-\Lambda_{n} x}, x>0, \operatorname{Re} \Lambda_{n}>0$, to the function $f$. These results involve comparisons between weighted $l^{p}$ sums of the sequence $\left(a_{n}\right)$ and weighted $L^{p}$ integrals of $f$ on $[0, \infty)$.

I. Introduction. In [6], Gaier proved that if

$$
f(x)=\sum_{n=1}^{\infty} a_{n} e^{-\Lambda_{n} x}, \quad 1 \leq \Lambda_{1}<\Lambda_{2}<\ldots,
$$

and $\Sigma \Lambda_{n}^{-1}$ converges, then

$$
\left|a_{n}\right| \leq 2 \Lambda_{n} p_{n} \int_{0}^{\infty}|f(x)| d x=2 \Lambda_{n} p_{n}\|f\|_{1}
$$

where $p_{n}=\Pi_{k \neq n}\left|\left(\Lambda_{k}+\Lambda_{n}\right) /\left(\Lambda_{k}-\Lambda_{n}\right)\right|$. (It is assumed that $\Sigma \Lambda_{n}^{-1}$ converges so that $p_{n}$ exists.) Anderson and Binmore [2] later proved that $\left|a_{n}\right| \leq$ $\left(2 \Lambda_{n}\right)^{1 / 2} p_{n}\|f\|_{2}$ and this was extended to

$$
\left|a_{n}\right| \leq\left(2 \Lambda_{n}\right)^{1 / p} p_{n}\|f\|_{p}, \quad 1 \leq p \leq 2,
$$

in [10]. All these results were superseded by Schwartz [9].

Recently, Binmore [3] has shown, among other things, that if $1 \leq \Lambda_{0} \leq$ $\Lambda_{1}<\Lambda_{2}<\cdots$, then

$$
\sum_{n=1}^{\infty}\left(\Lambda_{n}-\Lambda_{n-1}\right)\left|\frac{a_{n}}{\Lambda_{n} p_{n}}\right|^{2} \leq \pi\|f\|_{2}^{2}
$$

When $\left(\Lambda_{n}\right)$ is a lacunary sequence, i.e. $\Lambda_{n+1} \geq q \Lambda_{n}$ for some $q>1$, it is known [6] that $\left(p_{n}\right)$ is a bounded sequence, and so in this case (1.2) becomes

$$
\sum_{n=1}^{\infty}\left|a_{n}\right|^{2} \Lambda_{n}^{-1} \leq A(q) \int_{0}^{\infty}|f(x)|^{2} d x
$$

This is a special case of a result of Waterman [13], that if $\left(\Lambda_{n}\right)$ is lacunary, and $1 \leq \beta \leq p$, then

Received by the editors April 11, 1973.

AMS (MOS) subject classifications (1970). Primary 30A16, 30A18.

Key words and phrases. Carleson measure, exponential series. 


$$
\sum_{n=1}^{\infty}\left|a_{n}\right|^{p} \Lambda_{n}^{-\beta} \leq A(p, q, \beta) \int_{0}^{\infty}\left(1-e^{-x}\right)^{\beta-1}|f(x)|^{p} d x .
$$

It is the purpose of the present paper to extend the inequalities (1.1), (1.2), and (1.3) to cases where $\left(\Lambda_{n}\right)$ need not be real or lacunary, and $p \neq 2$.

Results on Carleson measures form the major tool here. A discussion of these may be found in Duren's book [5, Chapter IX]. The proofs found there are for the unit disc but may be readily adapted to the right half-plane, which will be denoted here by $\Pi^{+}$. The Hardy spaces of analytic functions $H^{r}$ will be with respect to $\Pi^{+}$. The result needed here is that if $\mu$ is a positive Borel measure in $\Pi^{+}$so that $\mu S_{h} \leq B h^{q / p}, 0<p \leq q<\infty$, whenever $S_{h}$ is a square of side $h$ in $\Pi^{+}$with one side on the $y$-axis, then

$$
\left(\int|F(z)|^{q} d \mu\right)^{1 / q} \leq C\|F\|_{H^{p}}
$$

where $C$ depends only on $B$. Thus, if $\left(\Lambda_{n}\right)$ is a sequence of points in $\Pi^{+}$ and if $\left(c_{n}\right)$ is a sequence of nonnegative constants so that $\Sigma_{\Lambda_{n} \epsilon s_{h}{ }^{c} n} \leq$ $B h^{q / p}$ then

$$
\left(\sum\left|F\left(\Lambda_{n}\right)\right|{ }^{q_{c}}\right)^{1 / q} \leq C\|F\|_{H^{p}} .
$$

Throughout this paper such a sequence will be called a Carleson measure, and it will be assumed that $1<p \leq q<\infty$ are fixed. This makes the statements of some of the theorems appear awkward since they are stated in terms of conjugate indices, but it seems to be the easiest way to handle things. It is important to emphasize that $C$ depends on $B$ alone and not on $\left(c_{n}\right)$, and this fact will be used in $\$ 3$. The constants $B$ and $C$ will carry this meaning throughout. Other constants will be denoted by $A(\alpha, \beta, \ldots)$ when they depend on parameters $\alpha, \beta, \ldots$. Conjugate indices are denoted by primes.

Throughout, let

$$
p_{n}=\prod_{k \neq n}\left|\frac{\Lambda_{k}+\bar{\Lambda}_{n}}{\Lambda_{k}-\Lambda_{n}}\right|
$$

when $\left(\Lambda_{n}\right)$ is a sequence of distinct points in $\Pi^{+}$. It is known that this product converges if and only if

$$
\sum \frac{\operatorname{Re} \Lambda_{n}}{1+\left|\Lambda_{n}\right|^{2}}<\infty,
$$

and unless otherwise stated, it will be assumed that (1.5) holds.

Let 


$$
W_{N}(z)=\prod_{1}^{N} \frac{z-\Lambda_{n}}{z+\bar{\Lambda}_{n}}
$$

Then $\left|W_{N}(i y)\right|=1$ and

$$
\left|W_{N}^{\prime}\left(\Lambda_{n}\right)\right|=\frac{1}{\left|2 \operatorname{Re} \Lambda_{n}\right|} \prod_{k \neq n}^{N}\left|\frac{\Lambda_{k}-\Lambda_{n}}{\Lambda_{k}+\bar{\Lambda}_{n}}\right| \geq \frac{1}{\left|2 \operatorname{Re} \Lambda_{n} p_{n}\right|}
$$

II. General results. To begi n, let

$$
F(z)=\sum_{1}^{N} \frac{a_{n}}{\left(z-\Lambda_{n}\right)^{m+1}} w_{N}(z)^{m+1} H(z)
$$

be a finite sum, where $m$ is a nonnegative integer, and $H \in H^{\infty}$. Then $F\left(\Lambda_{n}\right)=a_{n} W_{N}^{\prime}\left(\Lambda_{n}\right)^{m+1} H\left(\Lambda_{n}\right)$. It follows from (1.4) and (1.6) that

$$
\left(\sum_{1}^{N}\left|\frac{a_{n} H\left(\Lambda_{n}\right)}{\left(p_{n} \operatorname{Re} \Lambda_{n}\right)^{m+1}}\right|^{q} c_{n}\right)^{1 / q}
$$

$$
\leq 2^{m+1} C\left(\int_{-\infty}^{\infty}\left|\sum_{1}^{N} \frac{a_{n}}{\left(i t-\Lambda_{n}\right)^{m+1}} H(i t)\right|^{p} d t\right)^{1 / p}
$$

Inequalities of the type (2.1) are known to be equivalent to interpolation theorems. (See [4], for instance.) In this case (2.1) is equivalent to:

$$
\text { if } 1<p \leq q<\infty \text {, and } \sum\left|\frac{b_{n}\left(p_{n} \operatorname{Re} \Lambda_{n}\right)^{m+1}}{H\left(\Lambda_{n}\right) c_{n}^{1 / q}}\right|^{q^{\prime}}=S<\infty,
$$

then there is a $G \in L^{p^{\prime}}$ so that

$$
\int_{-\infty}^{\infty} \frac{G(i t) H(i t) d t}{\left(i t-\Lambda_{n}\right)^{m+1}} \doteq b_{n} \quad \text { and } \quad\|G\|_{p^{\prime}} \leq 2^{m+1} C S^{1 / q^{\prime}} .
$$

In particular, when $m=0$ and $H(z) \equiv 1,(2.2)$ shows that when $\left(\left(p_{n} \operatorname{Re} \Lambda_{n}\right)^{q}\right)$ is a Carleson measure then $\left(\Lambda_{n}\right)$ is an $\left(H^{p^{\prime}}, l^{q^{\prime}}\right)$ interpolating sequence [11].

In order to apply (2.1), the next theorem, on Fourier transforms, is needed.

(2.3) Theorem. Let $f$ be a continuous function with compact support in $R$, and let $F(x)=\int_{-\infty}^{\infty} f(t) e^{-i x t} d t$. If $1<p \leq q<\infty,-1 / q<\alpha \leq 0$, and $\beta=$ $1-(1 / p+1 / q)-\alpha \geq 0$ then

$$
\left(\int_{-\infty}^{\infty}|F(x)|^{q}|x|^{\alpha q} d x\right)^{1 / q} \leq A(p, q, \beta)\left(\int_{-\infty}^{\infty}|f(x)|^{p}|x|^{\beta p} d x\right)^{1 / p} .
$$


Thus the Fourier transform may be defined for all measurable functions $f$ for which the right-hand side is finite, and the inequality is still valid.

Proof. A theorem of Pitt [8] states that

$$
\left(\sum(|n|+1)-\beta p^{\prime}|\hat{f}(n)|^{p^{\prime}}\right)^{1 / p^{\prime}} \leq A(p, q, \beta)\left(\int_{-\pi}^{\pi}|f(\theta)|^{q^{\prime}}|\theta|^{-a q^{\prime}} d \theta\right)^{1 / q^{\prime}}
$$

when $1<q^{\prime} \leq p^{\prime}<\infty,-1 / q<\alpha \leq 0, \beta=1-(1 / p+1 / q)-\alpha \geq 0$. The dual result to this is that

$$
\left(\int_{-\pi}^{\pi}|f(\theta)|^{q}|\theta|^{\alpha q} d \theta\right)^{1 / q} \leq A(p, q, \beta)\left(\sum(|n|+1)^{\beta p}|\hat{f}(n)|^{p}\right)^{1 / p} .
$$

Now proceed as in [12, $\$ 3.2$ and 4.8].

Let $\lambda>0, a_{n}=a_{n}(\lambda)=\int_{n / \lambda}^{(n+1) / \lambda} f(x) d x, n=0, \pm 1, \cdots$, and $\Phi_{n}(x)=$ $\sum_{\nu=-n}^{n} a_{v} e^{i v x / \lambda}$. Then by (2.4)

$$
\begin{aligned}
\left(\int_{-\pi \lambda}^{\pi \lambda}\left|\Phi_{n}(x)\right|^{q}|x|^{q q} d x\right)^{1 / q} & =\left(\lambda^{1+a q} \int_{-\pi}^{\pi}\left|\sum_{-n}^{n} a_{v} e^{i v x}\right|^{q}|x|^{a q} d x\right)^{1 / q} \\
& \leq A(p, q, \beta) \lambda^{1 / q+a}\left(\sum(|v|+1)^{\beta p}\left|a_{v}\right|^{p}\right)^{1 / p}
\end{aligned}
$$

Moreover,

so that

$$
\begin{aligned}
\left|a_{n}\right|^{p} & \leq \lambda^{1-p} \int_{n / \lambda}^{(n+1) / \lambda}|f(x)|^{p} d x \\
& \leq A(|n|+1)^{-\beta_{p}} \lambda^{1-(1-\beta) p} \int_{n / \lambda}^{(n+1) / \lambda}|f(x)|^{p}|x|^{\beta p} d x
\end{aligned}
$$

$$
\left(\int_{-\pi \lambda}^{\pi \lambda}\left|\Phi_{n}(x)\right|^{q}|x|^{\alpha q} d x\right)^{1 / q} \leq A(p, q, \beta)\left(\int_{-\infty}^{\infty}|f(x)|^{p}|x|^{\beta p} d x\right)^{1 / p} .
$$

As in $[12, \S 3.2]$ it follows that the left-hand side tends to

$$
\left(\int_{-\infty}^{\infty}|F(x)|^{q}|x|^{a_{q}} d x\right)^{1 / q} \text { as } \lambda \rightarrow \infty .
$$

The first application of (2.3) is in conjunction with (2.1).

(2.5) Theorem. Let $1<p \leq q<\infty,-1 / q<\alpha \leq 0, \beta=1-2 / q-\alpha \geq 0$. If $f(x)=\Sigma a_{n} e^{-\Lambda_{n} x}$ is a finite sum then

$$
\left(\int_{0}^{\infty}|f(x)|^{p^{\prime}} x^{-\beta p^{\prime}} d x\right)^{1 / p^{\prime}} \leq A(\alpha, q)\left(\sum\left|a_{n}\left(1+\Lambda_{n}\right)-q\right| q^{\prime} c_{n}^{1-q^{\prime}}\right)^{1 / q^{\prime}} .
$$

Proof. To evaluate the left-hand side of the inequality, let $h(x)=$ $f(x) x^{-\beta}$. Since $p_{0}>1$, there is a function $k$ in $L^{p}(0, \infty)$ so that $\|k\|_{p} \leq 1$ and so that

$$
\|h\|_{p}{ }^{\prime}=\left|\int_{0}^{\infty} h(x) k(x) d x\right|=\left|\int_{0}^{\infty} f(x) k(x) x-\beta d x\right| .
$$

Let $g(x)=k(x) x^{-\beta}$. Then $\int_{0}^{\infty}|g(x)|^{p} x^{\beta p} d x \leq 1$ and 


$$
T=\left(\int_{0}^{\infty}|f(x)|^{p^{\prime}} x^{-\beta_{p^{\prime}}} d x\right)^{1 / p^{\prime}}=\left|\int_{0}^{\infty} \sum a_{n} e^{-\Lambda_{n} x} g(x) d x\right|=\left|\sum a_{n} G\left(\Lambda_{n}\right)\right| .
$$

By (1.4) and (2.3) (with $p=q$ )

$$
\begin{aligned}
\left(\sum\left|G\left(\Lambda_{n}\right)\left(1+\Lambda_{n}\right)^{\alpha}\right|^{q} c_{n}\right)^{1 / q} & \leq C\left(\int_{-\infty}^{\infty} \mid G(i t)(1+i t)^{\alpha \mid p} d t\right)^{1 / p} \\
& \leq C A(\alpha, p)\left(\int_{0}^{\infty}|g(x)|^{p}|x|^{\beta p} d x\right)^{1 / p}
\end{aligned}
$$

so that

$$
T \leq C A(\alpha, p)\left(\sum\left|a_{n}\left(1+\Lambda_{n}\right)^{-\alpha}\right|^{q^{\prime}} c_{n}^{1-q^{\prime}}\right)^{1 / q^{\prime}} .
$$

(2.6) Theorem. Let $1<p \leq q<\infty,-1 / p<\alpha \leq 0, \beta=1-2 / p-\alpha \geq 0$, and $m$ be a nonnegative integer. If $f(x)=\Sigma a_{n} e^{-\Lambda_{n} x}$ is a finite sum, then

$$
\left(\sum\left|\frac{a_{n}\left(1+\Lambda_{n}\right)^{a}}{\left(p_{n} \operatorname{Re} \Lambda_{n}\right)^{m+1}}\right|^{q} c_{n}\right)^{1 / q} \leq C A(p, \alpha, m)\left(\int_{0}^{\infty}|f(x)|^{p_{x}(m+\beta) p} d x\right)^{1 / p} .
$$

Proof. Since

$$
\int_{0}^{\infty} t^{m} f(t) e^{i x t} d t=(-1)^{m} m ! \sum \frac{a_{n}}{\left(i t-\Lambda_{n}\right)^{m+1}},
$$

the result follows immediately from (2.1) and (2.3), by letting $H(z)=(1+z)^{\alpha}$.

III. Applications. The problem of determining good inequalities relating series and integrals thus comes down to determining large Carleson measures on $\left(\Lambda_{n}\right)$. This is done here for some special cases. Throughout this section, $f, m, \beta$ and $\alpha$ will be assumed to possess the properties ascribed to them in (2.6).

First, if

$$
c_{n}=\left(\operatorname{Re} \Lambda_{n}\right)^{q / p}, \quad c_{k}=0, \quad k \neq n,
$$

then $\left(c_{n}\right)$ is a Carleson measure with $B=1$. Thus for each $n$

$$
\left|\frac{a_{n}\left(1+\Lambda_{n}\right)^{\alpha}}{\left(p_{n} \operatorname{Re} \Lambda_{n}\right)^{m+1}}\right|\left(\operatorname{Re} \Lambda_{n}\right)^{1 / p} \leq A(p, \beta, m)\left(\int_{0}^{\infty}|f|^{p} x^{(m+\beta) p}\right)^{1 / p} .
$$

Next, suppose $\left(\Lambda_{k}\right)$ to be a real increasing sequence, and set

$$
I_{k}=\left\{\Lambda_{n}: 2^{k-1} \leq \Lambda_{n}<2^{k}\right\}=\left\{\Lambda_{r_{k}}, \ldots, \Lambda_{s_{k}}\right\}, \quad k=1,2, \ldots .
$$

If $\left(n_{k}\right)$ is any subsequence of positive integers for which $\Lambda_{n_{k}} \in I_{k}$, set

$$
c_{n_{k}}=\Lambda_{s_{k}}^{q / p-1}\left(\Lambda_{s_{k}}-\Lambda_{s_{k-1}}\right)=\chi_{k}, \quad c_{n}=0, \quad n \neq n_{k} .
$$


362

W. T. SLED

Then when $2^{k-1}<h \leq 2^{k}$

$$
\sum_{\Lambda_{j} \in S_{h}} c_{j} \leq \sum_{j \leq k} \chi_{j} \leq \Lambda_{s_{k}}^{q / p} \leq 2^{k q / p} \leq(2 h)^{q / p}
$$

So $B=2^{q / p}$. Thus it follows from (2.6) that

$$
\begin{gathered}
\left(\sum \chi_{k} \sup \left\{\left|\frac{a_{n}\left(1+\Lambda_{n}\right)^{a}}{\left(p_{n} \operatorname{Re} \Lambda_{n}\right)^{m+1}}\right|^{q}: \Lambda_{n} \in I_{k}\right\}\right)^{1 / q} \\
\leq A(p, \beta, m)\left(\int_{0}^{\infty}|f(x)|^{p_{x}(m+\beta) p} d x\right)^{1 / p} .
\end{gathered}
$$

The sequence $c_{n}=\Lambda_{n}^{q / p-1}\left(\Lambda_{n}-\Lambda_{n-1}\right), n=1,2, \cdots$, gives the weaker inequality

$$
\begin{aligned}
\left(\sum\left|\frac{a_{n}\left(1+\Lambda_{n}\right)^{a}}{\left(p_{n} \operatorname{Re} \Lambda_{n}\right)^{m+1}}\right|^{q} \Lambda_{n}^{q / p-1}\left(\Lambda_{n}-\Lambda_{n-1}\right)\right)^{1 / q} \\
\leq A(p, \alpha, m)\left(\int_{0}^{\infty}|f(x)|^{p} x^{(m+\beta) p} d x\right)^{1 / p},
\end{aligned}
$$

and also a converse inequality, from (2.5),

$$
\begin{aligned}
& \left(\int_{0}^{\infty}\left|\sum a_{n} e^{-\Lambda_{n} x}\right|^{p^{\prime}} x^{-\beta p^{\prime}} d x\right)^{1 / p^{\prime}} \\
& \leq A(p, \alpha, m)\left(\sum \frac{\mid a_{n}\left(1+\Lambda_{n}\right)-q q^{\prime}}{\left(\Lambda_{n}^{q / p-1}\left(\Lambda_{n}-\Lambda_{n-1}\right)\right)^{\prime}-1}\right)^{1 / q^{\prime}}
\end{aligned}
$$

Owen [7] has shown that (3.4) holds even in $\beta>0$, when $p^{\prime}=q^{\prime}$.

Finally, let $\Lambda_{n}=1+i \lambda_{n}, n=\cdots-2,-1,0,1,2, \cdots$, and suppose that $0<\lambda_{n}-\lambda_{n-1} \leq 1$. If $c_{n}=\left(\lambda_{n}-\lambda_{n-1}\right)^{q / p}$ then $\left(c_{n}\right)$ is a Carlson measure. Then

$$
\begin{gathered}
\left(\sum\left|\frac{a_{n}\left(1+\Lambda_{n}\right)^{\alpha}}{\left(p_{n} \operatorname{Re} \Lambda_{n}\right)^{m+1}}\right|^{q}\left(\lambda_{n}-\lambda_{n-1}\right)^{q / p}\right)^{1 / q} \\
\leq A(p, a, m)\left(\int_{0}^{\infty}|f(x)|^{p} x^{(m+\beta) p} d x\right)^{1 / p}
\end{gathered}
$$

and

$$
\left(\int_{0}^{\infty}|f(x)|^{p^{\prime}} x-\beta p^{\prime} d x\right)^{1 / p^{\prime}} \leq A(p, a)\left(\sum \frac{\mid a_{n}\left(1+\Lambda_{n}\right)^{-a \mid q^{\prime}}}{\left(\lambda_{n}-\lambda_{n-1}\right)^{q / p\left(q^{\prime}-1\right)}}\right)^{1 / q^{\prime}}
$$


The remainder of this section is concerned with the relation of the inequalities (3.1)-(3.6) to known results.

The inequality (3.1) reduces to (1.1) when $\left(\Lambda_{n}\right)$ is a real sequence, $m=\beta=0$, and $1<p \leq 2$. However, when $2 \leq p$ the restriction that $p \beta \geq$ $p-2$ prevents the verification of the inequality

$$
\left|a_{n}\right| \leq A(p) \Lambda_{n}^{1 / p}\left|p_{n}\right|\|f\|_{p}, \quad 2 \leq p
$$

which had been conjectured in [2] and proved in some cases [1], [9]. This restriction on $\beta$ would seem at first glance to be due to $(2.3)$ and could perhaps then be removed by a different approach. But Dr. Anderson tells me that Professor D. J. Newman has shown that (3.7) is false and in fact that $\left|a_{n}\right| \leq A \Lambda_{n}^{1 / 2}\left|p_{n}\right|\|f\|_{\infty}$ is the best that can be hoped for.

The inequality (3.3) suffers from a similar defect, but where it is applicable it extends Binmore's and Waterman's results. In fact when $\Lambda=\left(\Lambda_{n}\right)$ is a lacunary real sequence then $\left|p_{n}\right| \leq A(\Lambda)[6]$, so

$$
\left(\sum \frac{\left|a_{n}\right|^{q} \Lambda_{n}^{q / p}}{\Lambda_{n}^{(m+1-a) q}}\right)^{1 / q} \leq A(p, \beta)\left(\int_{0}^{\infty}|f(x)|^{p} x^{(m+\beta) p} d x\right)^{1 / p},
$$

whereas Waterman's results require $0 \leq m+\beta \leq 1 / p^{\prime}$. And when $q=p=2$, $m=\beta=0$, (3.3) gives a rough form of Binmore's result (1.2).

Finally in (3.6), if $\lambda_{n}=n, s^{\prime}=q=p$, then it follows that

$$
\int_{0}^{2 \pi}\left|\sum a_{n} e^{-i n t}\right|^{s} t^{-\beta s} d t \leq A(p, \alpha)\left(\sum\left|a_{n}\right|^{s}(1+|n|)^{-\mu s}\right)
$$

if $0 \leq \beta<1 / s^{\prime}, \mu=1-2 / s^{\prime}-\beta \leq 0$. This is the dual to the result of Pitt mentioned in (2.3).

So the results seem to be sharp when measured against the existing literature. But the extent to which they are applicable is restricted.

\section{REFERENCES}

1. J. M. Anderson, Boundary properties of analytic functions with gap power series, Quart. J. Math. Oxford Ser. (2) 21 (1970), 247-256. MR 41 \#8677.

2. J. M.Anderson and K. G. Binmore, Coefficient estimates for lacunary power series and Dirichlet series. I, Proc. London Math. Soc. (3) 18 (1968), 36-48. MR 36 \#6624.

3. K. G. Binmore, Interpolation, approximation, and gap series, Proc. London Math. Soc. (3) 25 (1972), 751-768.

4. R. P. Boas, A general moment problem, Amer. J. Math. 63 (1941), 361-370. MR 2, 281.

5. P. L. Duren, Theory of $H^{p}$ spaces, Pure and Appl. Math., vol. 38, Academic Press, New York, 1970. MR 42 \#3552. 
6. D. Gaier, On the coefficients and the growth of gap power series, SIAM J. Numer. Anal. 3 (1966), 248-265. MR 34 \#4492.

7. P. M. Owen, A generalization of Hilbert's double series theorem, J. London Math. Soc. 5 (1930), 270-272.

8. H. R. Pitt, Theorems on Fourier and power series, Duke Math. J. 3 (1937), $747-755$.

9. L. Schwartz, Étude des sommes d'exponentielles réeles, Actualités Sci. Indust., no. 959, Hermann, Paris, 1943. MR 7, 294.

10. W. T. Sledd, Coefficient estimates for Dirichlet series, Trans. Amer. Math. Soc. 150 (1970), 69-76. MR $42 \# 3461$.

11. B. A. Taylor and D. L. Williams, Interpolation of $l^{q}$ sequences by $H^{p}$ functions, Proc. Amer. Math. Soc. 34 (1972), 181-186. MR $45 \# 3720$.

12. E. C. Titchmarsh, Introduction to the theory of Fourier integrals, 2nd ed., Clarendon Press, Oxford, 1948.

13. D. Waterman, On some high indices theorems, Trans. Amer. Math. Soc. 69 (1950), 468-478. MR 12, 496.

DEPARTMENT OF MATHEMATICS, MICHIGAN STATE UNIVERSITY, EAST LANSING, MICHIG AN 48824 